\title{
On the Relation Between Classical and Quantum Observables
}

\author{
Abhay Ashtekar \\ Département de Physique, Université de Clermont-Fd., F-63170 Aubière, France
}

\begin{abstract}
For systems with a finite number of degrees of freedom, the relation between classical and quantum observables is analysed. In particular, a precise statement of the correspondence limit is obtained.
\end{abstract}

\section{Introduction}

Consider a physical system with $n$ degrees of freedom. Let us suppose that in the (non-relativistic) classical description, one can introduce a smooth, $n$-dimensional, orientable manifold $\mathbf{C}$ (without boundary) to represent the configuration space of the system. Then, the cotangent bundle $\Gamma$ over $\mathbf{C}$ represents the phase space. This $\Gamma$ serves as the arena for classical mechanics: points of $\Gamma$ describe the permissible classical states while suitably regular functions on $\Gamma$ describe the classical observables. Dynamics manifests itself via a Hamiltonian vector field. The space of classical observables is endowed with two interesting mathematical structures. The first of these is the structure of an associative Abelian algebra, available on the space of functions on any manifold, while the second is the structure of a Lie algebra induced by the Poisson bracket which itself arises due to the presence of a natural symplectic structure on $\Gamma$.

In order to "quantize" this system, there is available the Dirac prescription [1]: Replace the Poisson brackets between classical observables by ( $\hbar / i$ times) the commutators of the corresponding quantum observables. Although the intuitive idea underlying this prescription is transparent, the precise "method of replacement" is not. What does "corresponding quantum observables" mean? Indeed, the well known factor ordering problems arise precisely because classical observables do not, in general, have unambiguous quantum analogues. For the class of systems described above, is there perhaps a universally available set of classical observables which does have its quantum analogue? More generally, what is the precise relation between classical and quantum observables? In the classical limit, does the commutator between two quantum operators vanish or is it related to the Poisson bracket between the corresponding classical observables? Is this cor- 
respondence well defined? That is, in the classical limit, does each quantum observable give rise, unambiguously, to a classical observable? What is the precise statement of the "classical limit" as far as the observables are concerned? Although these questions are rather elementary, and the answers probably well known among experts in geometrical quantum mechanics, to our knowledge, a detailed analysis of these issues - particularly of the mathematical structures involved - has not appeared in the literature ${ }^{1}$. The purpose of this note is to fill this gap.

\section{Schrödinger Quantum Description}

In this section, we briefly review ${ }^{2}$ a geometrical formulation of quantum kinematics. This discussion will also serve to fix notation to be used in the main analysis in Sect. 3 .

Consider the space $D$ of all complex valued, smooth scalar densities of weight $1 / 2$ and of compact support on the configuration space $\mathbf{C}$. This $D$ is a complex vector space. Next, introduce an inner product, $\langle$,$\rangle , on D$ as follows : Set $\langle\psi, \tilde{\psi}\rangle$ $=\int \psi^{*} \psi$, for all $\psi$ and $\tilde{\psi}$ in $D$ where the integral extends over all of $\mathbf{C}$ and where $\psi^{*}$ is the complex conjugate of $\psi$. (Note that we do not need any additional structure such as a metric or even an alternating tensor in order to define the integral; this is why we began with densities of weight $1 / 2$ rather than functions on $\mathbf{C}$.) The Cauchy completion $\bar{D}$ of the pre-Hilbert space $(D,\langle\rangle$,$) is the Hilbert space of$ quantum states. Next, we introduce certain operators which will represent, at the kinematic level, the basic quantum observables. Given a real-valued, smooth function $f$ of compact support on $\mathbf{C}$, define a configuration operator $\mathbf{Q}(f)$ as follows: $\mathbf{Q}(f) \psi:=f \cdot \psi$ for all $\psi$ in $\bar{D}$. Similarly, given a smooth vector field of compact support on $\mathbf{C}$, define a momentum operator $\mathbf{P}(V)$ as follows: $\mathbf{P}(V):=\hbar / i \mathscr{L}_{V} \psi$, for all $\psi$ in $D$, where $\mathscr{L}_{V}$ denotes the Lie derivative by the vector field $V$. Each $\mathbf{Q}(f)$ is a bounded self-adjoint operator on $\bar{D}$ while each $\mathbf{P}(V)$, with domain $D$, is an essentially self-adjoint operator on $\bar{D}$. The configuration and momentum operators have several interesting algebraic properties which will play an important role in our discussion of Sect. 3. First, they are linear in their arguments :

$$
\mathbf{Q}(f+r \tilde{f})=\mathbf{Q}(f)+r \mathbf{Q}(\tilde{f}) ; \quad \text { and }, \quad \mathbf{P}(V+r \tilde{V})=\mathbf{P}(V)+r \mathbf{P}(\tilde{V})
$$

for all scalar and vector fields $f, \tilde{f}$ and $V^{a}, \tilde{V}^{a}$ and for all real numbers $r$. Next, they satisfy the anti-commutation relations:

$$
\{\mathbf{Q}(f), \mathbf{Q}(\tilde{f})\}=2 \mathbf{Q}(f \cdot \tilde{f}) ; \quad \text { and }, \quad\{\mathbf{Q}(f), \mathbf{P}(V)\}=2 \mathbf{P}(f \cdot V)
$$

1 The text book treatments are generally incomplete and often misleading or even erroneous

2 For details, see, e.g., [2-4]. Note that in the present paper we are concerned not with the mathematical problems associated with the choice of a polarization on the phase space but rather with the relation between the classical and quantum observables of simple systems which already posses a natural vertical polarization 
and the canonical commutation relations:

$$
[\mathbf{Q}(f), \mathbf{Q}(\tilde{f})]=0 ; \quad[\mathbf{P}(V), \mathbf{Q}(f)] \subset \hbar / i \mathbf{Q}\left(\mathscr{L}_{V} f\right)
$$

and,

$$
[\mathbf{P}(V), \mathbf{P}(\tilde{V})]=\hbar / i \mathbf{P}\left(\mathscr{L}_{V} \tilde{V}\right),
$$

where we have set $\{A, B\}=A \cdot B+B \cdot A$ and $[A, B]=A \cdot B-B \cdot A$. Note that Planck's constant $\hbar$ appears only in Eq. (2). Hence one might expect Eq. (1) to hold, in an appropriate sense, in classical kinematics as well. We shall see in the next section that this expectation is correct. If $\mathbf{C}$ is chosen to be the Euclidean 3 -space, commutation relations (2) above imply, in particular, the usual canonical commutation relations between position and momentum operators as well as the commutation relations of these operators with the angular momentum operators ${ }^{3}$. Finally, note that in the above discussion only the geometrical structure naturally available on the manifold $\mathbf{C}$ has been used; in particular, no preferred choice of coordinates is necessary in the transition from classical to quantum description.

\section{Pre-observables, Quantization, and the Classical Limit}

In this section, we introduce a $*$-algebra $\mathscr{A}$ which, in a certain sense, encompasses the algebra of classical observables as well as that of quantum operators and facilitates the analysis of the relation between the two. In essence, one can regard $\mathscr{A}$ as the structure which synthesizes mathematical properties common to both classical and quantum observables, i.e., properties which are independent of the value of the Planck's constant $\hbar$. When the information on $\hbar$ is supplied, $\mathscr{A}$ admits a natural reduction to the $*$-algebra of configuration and momentum quantum operators introduced in Sect. 2 , while if $\hbar$ is set equal to zero, $\mathscr{A}$ reduces to the algebra of classical observables. Hence, elements of $\mathscr{A}$ ("self-adjoint" under the *-operation) will be referred to as "pre-observables". The introduction of the *-algebra of pre-observables serves two purposes: first, it rigorizes the Dirac prescription for passage from classical to quantum observables, and second, it makes transparent the mathematical structures involved in taking the classical limit.

Consider a smooth function $f$ of compact support on $\mathbf{C}$. Denote its pullback to $\Gamma$ by $q(f)$. Next, consider a smooth vector field $V^{a}$ on $\mathbf{C}$. In an obvious fashion this $V^{a}$ defines a function, linear in momentum, on $\Gamma$. Denote this function by $p(V)$. The $q^{\prime s}$ represent classical configuration observables while the $p^{\prime s}$, classical momentum observables. These observables may be regarded as the "basic" ones; sums of products of $q^{\prime s}$ and $p^{\prime s}$ generate the algebra of all classical observables. The space of $q^{\prime s}$ and $p^{\prime s}$ has a natural vector space structure. Denote this (real) vector space by $\mathbf{B}$ and its complexification by $\mathbf{B}_{c}$. The *-algebra of pre-observables will be constructed from this $\mathbf{B}_{c}$.

Let $\hat{\mathscr{A}}$ denote the tensor algebra over $\mathbf{B}_{c}: \hat{\mathscr{A}}:=\bigoplus_{k=0}^{\infty} \mathbf{B}_{c}^{\otimes k}$. Thus, a typical element $A$ of $\hat{\mathscr{A}}$ is a string with a finite number of non-zero entries, $A \equiv\left(v_{0}\right.$,

3 Choose for $f$ the coordinate functions $x, y$, and $z$, and for $V^{a}$, the Killing fields on Euclidean space. This of-course requires a trivial extension of our definitions of $\mathbf{Q}(f)$ and $\mathbf{P}(V)$ where the requirement of compact support on $f$ and $V$ are dropped 
$\left.v_{1}, \ldots, v_{n}, \ldots, 0,0, \ldots\right)$, where $v_{0}$ denotes a complex number and $v_{n}$ a $n$-th rank (contravariant) tensor over $\mathbf{B}_{c}$. The set of these strings has a natural vector space structure. The associative product is defined in an obvious way: If $A$ is as above and $B=\left(w_{0}, w_{1}, \ldots, w_{k}, 0,0, \ldots\right)$, then $A \cdot B=\left(v_{0} w_{0}, v_{0} w_{1}+w_{0} v_{1}, \ldots, v_{0} w_{m}\right.$ $\left.+v_{1} \otimes w_{m-1}+\ldots+v_{m-1} \otimes w_{1}+w_{0} v_{m}\right)$. Thus, $\hat{\mathscr{A}}$ is an associative algebra. Next, introduce a *operation on $\hat{\mathscr{A}}$ as follows: Demand that the action of the *-operation on $\mathbf{B}_{c}$ be the complex conjugation and extend its action to all of $\hat{\mathscr{A}}$ by requiring anti-linearity, i.e., $(\lambda A+\mu B)^{*}=\lambda^{*} A^{*}+\mu^{*} B^{*}$, and the product rule, $(A B)^{*}$ $=B^{*} \cdot A^{*}$, for all $A$ and $B$ in $\hat{\mathscr{A}}$ and complex numbers $\lambda$ and $\mu$, where $\lambda^{*}$ denotes the complex conjugate of $\lambda$. Then it is easy to verify that the *-operation automatically satisfies $\left(A^{*}\right)^{*}=A$; it is thus an involution on the algebra $\hat{\mathscr{A}}$. To obtain the *-algebra $\mathscr{A}$, we need to introduce on $\hat{\mathscr{A}}$ conditions analogous to Eq. (1b). Recall that each basic classical observable $q(f)$ and $p(V)$ belongs to $\mathbf{B}$ and hence also to $\mathbf{B}_{c}$. Consider the *-ideal $I$ of $\hat{\mathscr{A}}$ generated by elements of the type $(0$, $2 q(f \cdot \tilde{f})+2 p(g V), q(f) \otimes q(\tilde{f})+q(\tilde{f}) \otimes q(f)+q(g) \otimes p(V)+p(V) \otimes q(g), 0,0,0, \ldots, 0, \ldots)$ where $f, \tilde{f}$, and $g$ are arbitrary smooth scalar fields, and $V^{a}$, an arbitrary vector field, of compact support on $\mathbf{C}$. Set $\mathscr{A}=\hat{\mathscr{A}} / I$. This $\mathscr{A}$ is the required *-algebra. An element $A$ of $\mathscr{A}$ will be called a pre-observable if $A=A^{*}$.

It is easy to verify that each $q(f)$ and $p(V)$ gives rise, unambiguously, to an element of $\mathscr{A}$ : Since the only element of $\hat{\mathscr{A}}$ of the type $(0, v, 0, \ldots, 0, \ldots)$ which belongs to $I$ is the zero element, two distinct elements of $B$ can not find themselves in the same equivalence class after the quotient operation. Denote by $Q(f)$ the element of $\mathscr{A}$ corresponding to $q(f)$ and by $P(V)$ the element corresponding to $p(V)$. Then, by the very construction of $\mathscr{A}$ it follows that $Q^{\prime s}$ and $P^{\prime s}$ satisfy Eq. (1a, b): (1a) is "built in to" the introduction of $\hat{\mathscr{A}}$ as the tensor algebra over $B_{c}$ while (1b) is incorporated via the operation of taking quotient of $\hat{\mathscr{A}}$ by $I$. Thus, $Q^{\prime s}$ and $P^{\prime s}$ satisfy the usual algebraic relations between quantum configuration and momentum operators except ofcourse the canonical commutation relations which require the knowledge of $\hbar$. Hence, $Q^{\prime s}$ and $P^{\prime s}$ may be regarded as the basic preobservables.

Next, we introduce a Lie bracket on $\mathscr{A}$. The key idea is to exploit the fact that the vector space $\mathbf{B}$, regarded as a sub-space of the space of all classical observables is invariant under the operation of taking Poisson brackets: We have

$$
[q(f)+p(V), q(\tilde{f})+p(\tilde{V})]_{\text {P.B. }}=q\left(\mathscr{L}_{V} \tilde{f}-\mathscr{L}_{\tilde{V}} f\right)+p\left(\mathscr{L}_{V} \tilde{V}\right)
$$

for all $f, \tilde{f}$ and $V^{a}, \tilde{V}^{a}$. This enables us to introduce a Lie bracket, $[,]_{L}$, on $\mathscr{A}$ as follows. Set

$$
[Q(f)+P(V), Q(\tilde{f})+P(\tilde{V})]_{L}=Q\left(\mathscr{L}_{V} \tilde{f}-\mathscr{L}_{\tilde{V}} f\right)+P\left(\mathscr{L}_{V} \tilde{V}\right)
$$

and require, for all $A, B, C$ in $\mathscr{A}$ and complex numbers $\lambda$, that the following conditions hold:

$$
\begin{aligned}
{[A, B]_{L} } & =-[B, A]_{L}, \\
{[A+\lambda B, C]_{L} } & =[A, C]_{L}+\lambda[B, C]_{L}, \\
{[A B, C]_{L} } & =A \cdot[B, C]_{L}+[A, C]_{L} \cdot B .
\end{aligned}
$$


We claim that Eqs. $(4 \mathrm{a}-\mathrm{d})$ unambiguously define a Lie bracket on $\mathscr{A}$. This $[,]_{L}$ is, furthermore, a natural extension to $\mathscr{A}$ of the Poisson bracket on $\mathbf{B}$.

Thus, $\mathscr{A}$ now has the structure of an associative non-Abelian $*$-algebra, as well as that of a Lie algebra. In the introduction of these structures, we have used only those properties of the given system which are associated invariantly with the presence of a natural (classical) configuration manifold $\mathbf{C}$.

So far, the two structures on $\mathscr{A}$ are quite independent of each other: the Lie bracket $[,]_{L}$ is in no way related to the commutator bracket of the associative product. "Quantization", from an algebraic point of view, consists of introducing relations between the two structures. Consider the ${ }^{*}$-ideal $\mathscr{I}_{\hbar}$ of $\mathscr{A}$ generated by elements of the type $A \cdot B-B \cdot A-\hbar / i[A, B]_{L}$ where $A$ and $B$ are arbitrary elements of $\mathscr{A}$ and $\hbar$ any fixed real number. Set $\mathscr{A}_{\hbar}=\mathscr{A} / \mathscr{I}_{\hbar}$. Then $\mathscr{A}_{\hbar}$ is a ${ }^{*}$-algebra. Furthermore, it is straightforward to verify that $\mathscr{A}_{h}$ is in fact naturally isomorphic to the *-algebra generated by the operators $\mathbf{Q}(f)$ and $\mathbf{P}(V)$ (together with the identity operator on $D$ ) introduced in the previous section ${ }^{4}$. (Here, we consider $\mathbf{Q}(f)$ and $\mathbf{P}(V)$ as having the common domain in $D$. The isomorphism sends the equivalence class to which $Q(f)$ [resp. $P(V)$ ] in $\mathscr{A}$ belongs to the operator $\mathbf{Q}(f)$ [resp. $\mathbf{P}(V)$ ] on D.) Thus, $\mathscr{A}_{\hbar}$ is precisely the ${ }^{*}$-algebra of quantum operators! ${ }^{5}$ The Dirac prescription of setting the commutator equal to ( $\hbar / i$ times) the Lie bracket is indeed

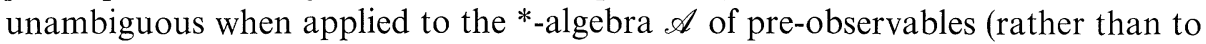
the algebra of classical observables). Finally, note that while $\mathscr{A}$ is equipped with two distinct brackets - the commutator and the bracket $[,]_{L}-\mathscr{A}_{\hbar}$ inherits only one : it is precisely the quotient operation needed in the transition from pre-observables to quantum operators which identifies the two brackets.

What happens when $\hbar$ is set equal to zero? The ideal $\mathscr{I}_{0}$ is generated by elements of $\mathscr{A}$ of the type $A \cdot B-B \cdot A$. Hence, $\mathscr{A}_{0}=\mathscr{A} / \mathscr{I}_{0}$ is an Abelian, associative *-algebra. Again, it is easy to verify ${ }^{4}$ that $\mathscr{A}_{0}$ is naturally isomorphic to the algebra of complex-valued smooth functions on $\Gamma$ which are polynomials in momenta and are such that the projection to $\mathbf{C}$ of their support is of compact closure. [Ofcourse, the isomorphism sends the equivalence class to which $Q(f)$ in $\mathscr{A}$ belongs to the classical observable $q(f)$, and similarly for $P(V)$.] Furthermore, the *-operation on $\mathscr{A}$ corresponds to the complex-conjugation operation on the space of functions on $\Gamma$. Thus, $\mathscr{A}_{0}$ is just the complexification of the algebra of classical observables. What is the status of the Lie bracket $[,]_{L}$ ? Now, since $\hbar$ is set equal to zero, the commutator and the Lie bracket $[,]_{L}$ are not identified in the quotient construction. Furthermore, this bracket does give rise to an unambiguous Lie bracket on the ${ }^{*}$-algebra $\mathscr{A}_{0}$. (That is, $\mathscr{I}_{0}$ turns out also to be a Lie-ideal on $\mathscr{A}$.) This Lie bracket on $\mathscr{A}_{0}$ is precisely the (image under the natural isomorphism, just referred to, of the) Poisson bracket on the space of functions on $\Gamma !^{4}$ Thus, the quotient operation is such that, for any non-zero value of $\hbar, \mathscr{A}_{\hbar}$ inherits only one of the two brackets - or, equivalently, Lie algebra structures - of $\mathscr{A}$, while $\mathscr{A}_{0}$ inherits them both; the commutator bracket induces a trivial Lie-algebra structore on $\mathscr{A}_{0}$ and the Lie bracket $[,]_{L}$ induces the Poisson bracket.

4 Proofs of these assertions are omitted because they are straightforward and long 5 Indeed, using $\mathscr{A}_{\hbar}$ one can construct the Hilbert space of states via the Gelfand-Naimark-Segal [5] construction 
To summarize, both quantum and classical observables arise as equivalence classes of pre-observables: $\mathscr{A}_{\hbar}=\mathscr{A} / \mathscr{I}_{\hbar}$ and $\mathscr{A}_{0}=\mathscr{A} / \mathscr{I}_{0}$. However, since the ideals involved in the two quotients are distinct from each other, there is no simple relation between the two sets of equivalence classes (see Fig. 1). In particular, there

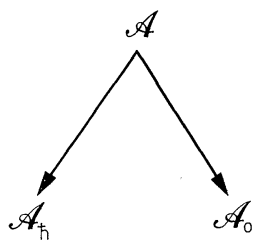

Fig. 1. $\mathscr{A}_{\hbar}=\mathscr{A} / \mathscr{I}_{\hbar}$ and $\mathscr{A}_{0}=\mathscr{A} / \mathscr{I}_{0}$. Hence, although there exists a natural $*$-algebra homeomorphism from $\mathscr{A}$ on to $\mathscr{A}_{\hbar}$ as well as on to $\mathscr{A}_{0}$, there does not exist a natural mapping from $\mathscr{A}_{\hbar}$ to $\mathscr{A}_{0}$ or vice versa

is no natural mapping from $\mathscr{A}_{0}$ to $\mathscr{A}_{\hbar}$ or conversely from $\mathscr{A}_{\hbar}$ to $\mathscr{A}_{0}$ : just as classical observables do not, in general, have unambiguous quantum analogues, quantum observables do not, in general, have unambiguous classical analogues. Nonetheless the correspondence limit can be expressed by noting that for each real number $\hbar, \mathscr{A}_{\hbar}=\mathscr{A} / \mathscr{I}_{\hbar}$ is a ${ }^{*}$-algebra; $\mathscr{A}_{0}$ is the (complexified) algebra of classical observables and when $\hbar$ has the value of Planck's constant, $\mathscr{A}_{\hbar}$ is the algebra of quantum operators. This formulation has the advantage that, at the algebraic level, it is easy to see what is preserved in the classical limit and what is not since all observables, quantum as well as classical, are obtained from a single structure.

Acknowledgements. I am grateful to Robert Geroch, Pong-Soo Jang, and Anne Magnon-Ashtekar for discussions.

\section{References}

1. Dirac, P.A.M.: Principles of quantum mechanics. London: Clarendon 1930

2. Mackey, G. : Mathematical foundations of quantum mechanics. New York: Benjamin 1963

3. Geroch, R.: Geometrical quantum mechanics. University of Chicago lecture notes 1974 (unpublished)

4. Simms, D.J., Woodhouse, N.M.J.: Lectures on geometric quantization. Berlin, Heidelberg, New York: Springer 1976

5. Gelfand, I., Naimark, M.: Sobernik 12 (1943);

Segal, I. : Bul. Am. Math. Soc. 53 (1947)

Communicated by R. Geroch

Received May 31, 1979 